\section{OP34 FINDING WHAT MATTERS: USING NATURAL LANGUAGE PROCESSING TO IDENTIFY PATIENT CARE PREFERENCES WITHIN CLINICAL NOTES}

${ }^{1} S$ Zupanc ${ }^{*},{ }^{2} \mathrm{~K}$ Lee, ${ }^{1} \mathrm{E}$ Moseley, ${ }^{1} \mathrm{Y}$ Yeh, ${ }^{1} \mathrm{C}$ Lindvall. ${ }^{1}$ Dana-Farber Cancer Institute, Boston, USA; ${ }^{2}$ Brigham and Women's Hospital, Boston, USA

\subsection{6/spcare-2019-ACPICONGRESSABS.34}

Background Delivering care that is consistent with patient preferences is considered the outcome of successful advance care planning interventions. However, patient preferences are often difficult to ascertain within clinical notes, and thus difficult to extract and utilize in clinical or evaluative settings. The objective of this study is to show the efficiency and accuracy of two natural language processing (NLP) methods in identifying documentation within the free-text of clinical notes.

Methods Rule-based and machine learning NLP methods were developed and trained on a dataset of 449 clinical notes derived from Multi Parameter Intelligent Monitoring of Intensive Care (MIMIC) III database. Human annotators identified instances of code status limitation and patient care preference documentation in a second validation dataset of 192 clinical notes. We then assessed the performance of the rule-based and machine learning NLP mathods in identifying code status limitation and patient care preference documentation in the validation dataset.

Results Machine learning NLP identified documentation with a sensitivity ranging from $85.1-98.3 \%$ and a specificity ranging from 91.0\%-97.0\%. Performance of rule-based NLP was comparable, identifying documentation of code status limitation with a sensitivity of $98.3 \%$ and a specificity of $97.7 \%$ and patient care preferences with a sensitivity of $81.5 \%$ and a specificity of $83.0 \%$.

Conclusions NLP methods are reliable tools for identifying information related to patient care preferences within clinical notes. Machine learning NLP may be better suited to identify documentation of conversations that vary in the way they are recorded, such as conversations related to goals of care.

\section{OP35 A NATIONAL FRAMEWORK FOR ANTICIPATION IN HEALTH CARE: THE SWISS PROPOSAL}

${ }^{1} \mathrm{~S}$ Eychmueller*, ${ }^{1} \mathrm{~S}$ Felber, ${ }^{2} \mathrm{~T}$ Krones. ${ }^{1}$ University of Bern, Bern, Switzerland; ${ }^{2}$ University of Zurich, Zurich, Switzerland

\subsection{6/spcare-2019-ACPICONGRESSABS.35}

Background Anticipatory planning including advance care planning has become one of the megatrends in health care. As in many countries, terminology and understanding of anticipatory planning varies significantly.

Aim For a common understanding of anticipation and guidance in clinical practice, we aimed to develop a national framework for anticipation in health care in Switzerland.

Method we undertook a national consensus project with representatives from all cultural regions in Switzerland. After review of the literature and existing guidelines and/or recommendations we performed a three Delphi process including additional national expert review.

Results We propose a three level model of anticipatory planning in health care. Level one aims to initiate general anticipatory planning including discussion on individual values, preferences and expectations; experts from various professional background (e.g. pastoral care, social care, psychology) may offer support on this level. Level two focuses on disease specific anticipatory care planning; expertise in regard of potential complication of specific diagnosis/disease trajectory is necessary to provide assistance while planning. Level three finally is dedicated to advance care planning (ACP) for clinical situations without capacity for judgement; guidance by professionals with a background in intensive and emergency care may be needed.

Conclusions a national consensus process on definitions of various levels for a participatory approach in anticipatory planning helps to create a solid basis for common understanding of anticipation in health care, to highlight professional roles and expertise for counselling on each level, and to prevent low quality in written documentation.

\section{OP36 PREVALENCE OF ADVANCE CARE DIRECTIVES AMONG OLDER AUSTRALIANS ACCESSING HEALTH AND RESIDENTIAL AGED CARE SERVICES: MULTI-CENTRE AUDIT STUDY}

${ }^{1} \mathrm{~L}$ Nolte ${ }^{*},{ }^{1} \mathrm{~K}$ Buck, ${ }^{2} \mathrm{R}$ Ruseckaite, ${ }^{1} \mathrm{H}$ Kelly, ${ }^{1} \mathrm{M}$ Sellars, ${ }^{3} \mathrm{C}$ Sinclair, ${ }^{4} \mathrm{~J}$ Clayton, ${ }^{1} \mathrm{~K}$ Detering. ${ }^{1}$ Austin Health, Heidelberg, Australia; ${ }^{2}$ Monash University, Melbourne, Australia; ${ }^{3}$ University of Western Australia, Albany, Australia; ${ }^{4}$ Greenwich Hospital and University of Sydney, Sydney, Australia

\subsection{6/spcare-2019-ACPICONGRESSABS.36}

Background Advance care planning (ACP) is a priority in Australian health policy, legislation and accreditation standards. ACP supports people to consider and communicate their future treatment preferences and document them in an advance care directive (ACD). However, the availability of ACDs at the point of care amongst Australians is unknown. The aim of this study was to describe the prevalence of ACDs in those aged $\geq 65$ years accessing general practice (GP), hospitals and residential aged care facilities (RACF).

Methods A prospective multi-centre health record audit. Literature review informed the methodology. Auditors received education and jurisdictional-specific audit manuals. Recruitment of organisations was via expression of interest. The primary outcome was presence of an ACD.

Results Fifty-one sites participated (13 GPs, 12 hospitals, 26 RACFs), representing six jurisdictions. 2,285 health records were audited; 503 attending GPs, 574 in hospitals, and 1,208 in RACFs. $30 \%$ of people had at least one ACD. Most (21\%) were non-statutory documents. The prevalence of statutory ACD-preferences for care was 3\%; the prevalence of statutory ACD-substitute decision-maker was $11 \%$. ACD prevalence in GP was low (3\%) compared to hospitals (16\%) and RACFs (48\%).

Conclusions Approximately 30\% of older Australians had at least one ACD in their health record, and the majority of these were non-statutory ACDs. Priorities to increase accessibility of documentation may include improved policy, promotion of ACD uptake amongst older persons, storage within health record systems, workforce education and training, information resources, and ongoing prevalence monitoring. These initiatives are required across all sectors, especially GP. 